\title{
CAPILLARY EFFECT ON VERTICALLY EXCITED SURFACE WAVE IN CIRCULAR CYLINDRICAL VESSEL *
}

\author{
JIAN Yong-jun (菅永军 $)^{1,2}, \quad$ E Xue-quan (鄂学全) $)^{2}, \quad$ ZHANG Jie (张杰) $)^{1,3}$ \\ (1. First Institute of Oceanography, State Oceanic Administration, \\ Qingdao 266061, Shandong Province, P. R. China; \\ 2. Institute of Mechanics, Chinese Academy of Sciences, Beijing 100080, P. R. China; \\ 3. Key Laboratory of Marine Science and Numerical Modeling, State Ocean Administration, \\ Qingdao 266061, Shandong Province, P. R. China) \\ (Communicated by DAI Shi-qiang)
}

\begin{abstract}
In a vertically oscillating circular cylindrical container, singular perturbation theory of two-time scale expansions was developed in inviscid fluids to investigate the motion of single free surface standing wave including the effect of surface tension. A nonlinear slowly varying amplitude equation, which incorporates cubic nonlineas. term, external excitation and the influence of surface tension, was derived from potential flow equation. The results show that, when forced frequency is lower, the sffect cf suríace tension on mode selection of surface wave is not important. Hows rer, when forcod frequicncy is higher, the surface tension can not be neglected. This provad that the surface tension causes free surface returning to equilibrium !ccation In addition, due to considering the effect of surface tension, the theoritical result approarhis to experimental results much more than that of no surface tension.
\end{abstract}

Key words: 'ercically forced oscillation; nonlinear amplitude equation; surface tension; surface wave modes

Chinese livitrary Classification: O353.2

2000 Mathematics Subject Classification: 76B15; 76B07; 76M45

Digital Object Identifier(DOI): 10.1007/s 10483-006-0211-1

\section{Introduction}

Since the 1980 s, the onset of the instability of surface waves in a vertically excited cylindrical container ${ }^{[1]}$, secondary instabilities and transition to spatio-temporal chaos ${ }^{[2,3]}$ and turbulence ${ }^{[4]}$ were extensively investigated for weakly viscous fluids. E Xuequan et al ${ }^{[5-7]}$ carried out the flow visualization and experimental study on surface wave patterns in a circular cylindrical vessel by vertical external vibrations. They obtained very beautiful photographs of free surface patterns, and most of them have not been reported before. Recently, a theoretical treatment associated with the experiments in Refs.[5-7] was established by Jian ${ }^{[8-10]}$, from which the nonlinear amplitude equation without considering the effect of surface tension and second order free surface displacements were obtained by two-time scale singular perturbation expansion in ideal fluids. Although theoretical contours of free surface waves agree well with the experimental visualization, the forced frequency had large differences.

In this article, a nonlinear amplitude equation, which is similar to that of Ref.[8], is obtained from Euler equation in inviscid fluid including the effect of surface tension. The difference

* Received Jun.9, 2004; Revised Aug.17, 2005

Project supported by the National Natural Science Foundation of China (Nos.19772063 and 19772068)

and the Doctoral Research Fund of the Ministry of Education (No.20010141024)

Corresponding author JIAN Yong-jun, Doctor, E-mail:jianyongjun@yahoo.com.cn 
of two amplitude equations is that the latter includes the effect of surface tension. Finally, some theoretical results with surface tension are compared with those without surface tension. It showed that theoretical forced frequency with the effect of surface tension much closes to experimental results when the same pattern is excited.

\section{Governing Equations and Non-dimensional Formulation}

We consider surface waves excited by the vertical motion of a circular cylindrical basin filled with fluid. The physical modeling is the same as Fig.1 in Ref.[8]. The meanings of parameters are the same as those in Ref.[8]. It is assumed that the fluid is inviscid, incompressible and the motion is irrotational, there must exist a velocity potential function $\phi(r, \theta, z, t)$, and satisfy the following governing equation:

$$
\frac{\partial^{2} \phi}{\partial r^{2}}+\frac{1}{r} \frac{\partial \phi}{\partial r}+\frac{1}{r^{2}} \frac{\partial^{2} \phi}{\partial \theta^{2}}+\frac{\partial^{2} \phi}{\partial z^{2}}=0, \quad 0<r<R, \quad-h \leq z \leq \eta(r, \theta, t) .
$$

The kinetic and kinematic boundary conditions at free surface $z=\eta(r, \theta, t)$ are

$$
\begin{aligned}
& \frac{\partial \phi}{\partial t}+\frac{1}{2}\left[\left(\frac{\partial \phi}{\partial r}\right)^{2}+\frac{1}{r^{2}}\left(\frac{\partial \phi}{\partial \theta}\right)^{2}+\left(\frac{\partial \phi}{\partial z}\right)^{2}\right] \\
& +\left(g-\ddot{z}_{0}\right) \eta-\frac{\Gamma}{\rho}\left(\frac{\partial^{2} \eta}{\partial r^{2}}+\frac{1}{r} \frac{\partial \eta}{\partial r}+\frac{1}{r^{2}} \frac{\partial^{2} \eta}{\partial \theta^{2}}\right)=0 \\
& \frac{\partial \eta}{\partial t}+\frac{1}{r^{2}} \frac{\partial \phi}{\partial \theta} \frac{\partial \eta}{\partial \theta}+\frac{\partial \phi}{\partial r} \frac{\partial \eta}{\partial r}-\frac{\partial \phi}{\partial z}=0
\end{aligned}
$$

where constants $\Gamma$ and $\rho$ denote the surface tension coefifient and the dcisity of the fluid, respectively. The effect of surface tension is irircduced by bonidary condition of the free surface. In addition, since the infweure of viscosity is : onored, the boundary conditions on the side-wall and at the bottom of the vessel berome zero normal velocity for rigid container, namely,

$$
\begin{aligned}
& \frac{\partial \phi}{\partial r}=0, \quad r=R, \\
& \frac{\partial \phi}{\partial z}=0, \quad z=-h .
\end{aligned}
$$

Taking the radius $R$ of the vessel as the length scale, and nondimensionalizing all related independent and unknown variables, the following scalings are adopted:

$$
\begin{aligned}
& z^{*}=z / R, r^{*}=r / R, \eta^{*}=\eta / R, t^{*}=t / \sqrt{R / g}, \phi^{*}=\phi /(R \sqrt{g R}), \\
& A^{*}=A / R, \omega_{0}^{*}=\omega_{0} / \sqrt{g / R}, \varepsilon^{*}=4 A \omega_{0}^{2} / g, \Gamma^{*} / \rho^{*}=\left(g R^{2}\right) \Gamma / \rho .
\end{aligned}
$$

Note that the asterisks " $*$ " denote dimensionless quantities and are subsequently dropped. Substituting Eq.(6) into Eqs.(1)-(5), then expanding Eqs.(2) and (3) into Taylor series at $z=0$ by neglecting the term $O\left(\varepsilon^{4}\right)$, we obtain the following non-dimensional governing equation:

$$
\frac{\partial^{2} \phi}{\partial r^{2}}+\frac{1}{r} \frac{\partial \phi}{\partial r}+\frac{1}{r^{2}} \frac{\partial^{2} \phi}{\partial \theta^{2}}+\frac{\partial^{2} \phi}{\partial z^{2}}=0, \quad 0<r<1, \quad-h / R<z<0 .
$$

Boundary conditions at free surface $z=0$,

$$
\begin{aligned}
& \frac{\partial \phi}{\partial t}+\frac{1}{2}\left[\left(\frac{\partial \phi}{\partial r}\right)^{2}+\frac{1}{r^{2}}\left(\frac{\partial \phi}{\partial \theta}\right)^{2}+\left(\frac{\partial \phi}{\partial z}\right)^{2}\right]+\left[1+\varepsilon^{2} \cos \left(2 \omega_{0} t\right)\right] \eta \\
& -\frac{\Gamma}{\rho}\left(\frac{\partial^{2} \eta}{\partial r^{2}}+\frac{1}{r} \frac{\partial \eta}{\partial r}+\frac{1}{r^{2}} \frac{\partial^{2} \eta}{\partial \theta^{2}}\right)+\frac{\partial^{2} \phi}{\partial t \partial z} \eta
\end{aligned}
$$




$$
\begin{aligned}
& +\left(\frac{\partial \phi}{\partial r} \frac{\partial^{2} \phi}{\partial r \partial z}+\frac{1}{r^{2}} \frac{\partial \phi}{\partial \theta} \frac{\partial^{2} \phi}{\partial \theta \partial z}+\frac{\partial \phi}{\partial z} \frac{\partial^{2} \phi}{\partial z^{2}}\right) \eta+\frac{1}{2} \frac{\partial^{3} \phi}{\partial t \partial z^{2}} \eta^{2}=0 \\
& \frac{\partial \eta}{\partial t}-\frac{\partial \phi}{\partial z}+\frac{\partial \phi}{\partial r} \frac{\partial \eta}{\partial r}+\frac{1}{r^{2}} \frac{\partial \phi}{\partial \theta} \frac{\partial \eta}{\partial \theta}-\frac{\partial^{2} \phi}{\partial z^{2}} \eta \\
& +\frac{\partial^{2} \phi}{\partial r \partial z} \eta \frac{\partial \eta}{\partial r}+\frac{1}{r^{2}} \frac{\partial^{2} \phi}{\partial \theta \partial z} \eta \frac{\partial \eta}{\partial \theta}-\frac{1}{2} \frac{\partial^{3} \phi}{\partial z^{3}} \eta^{2}=0
\end{aligned}
$$

Boundary conditicis on side-wall and at the bottom,

$$
\begin{array}{ll}
\frac{\partial \phi}{\partial r}=0, & r=1, \\
\frac{\partial \phi}{\partial z}=0, & z=-h / R .
\end{array}
$$

In order to solve Eqs.(7)-(11) by the two-time scale perturbation expansion, a slowly varying time scale $\tau$ is introduced, and let $\tau=\varepsilon^{2} t$, we have

$$
\frac{\partial}{\partial t}=\frac{\partial}{\partial t}+\varepsilon^{2} \frac{\partial}{\partial \tau}+\cdots
$$

To seek the solutions of $\phi(r, \theta, z, t)$ and $\eta(r, \theta, t)$, we expand them in a power series in $\varepsilon$ as

$$
\left\{\begin{array}{l}
\phi(r, \theta, z, t, \tau)=\varepsilon \phi_{1}+\varepsilon^{2} \phi_{2}+\varepsilon^{3} \phi_{3}+\cdots \\
\eta(r, \theta, t, \tau)=\varepsilon \eta_{1}+\varepsilon^{2} \eta_{2}+\varepsilon^{3} \eta_{3}+\cdots
\end{array}\right.
$$

Substituting Eqs.(12) and (13) into the non-dimensional rquations (7)-(ii), we can give the approximate equations by comparing the coeficients of the sruel! parameter $\varepsilon^{i}$ at the two sides of the equations.

\section{First Ordor Approximate Solutions}

The governisg equation and boundary conditions of first order problem are

$$
\begin{aligned}
& \frac{\partial^{2} \phi_{1}}{\partial r^{2}}+\frac{1}{r} \frac{\partial \phi_{1}}{\partial r}+\frac{1}{r^{2}} \frac{\partial^{2} \phi_{1}}{\partial \theta^{2}}+\frac{\partial^{2} \phi_{1}}{\partial z^{2}}=0, \quad 0<r<1, \quad-h / R<z<0, \\
& \frac{\partial \phi_{1}}{\partial t}+\eta_{1}-\frac{\Gamma}{\rho}\left(\frac{\partial^{2} \eta_{1}}{\partial r^{2}}+\frac{1}{r} \frac{\partial \eta_{1}}{\partial r}+\frac{1}{r^{2}} \frac{\partial^{2} \eta_{1}}{\partial \theta^{2}}\right)=0, \quad z=0, \\
& \frac{\partial \eta_{1}}{\partial t}-\frac{\partial \phi_{1}}{\partial z}=0, \quad z=0, \\
& \frac{\partial \phi_{1}}{\partial r}=0, \quad r=1, \\
& \frac{\partial \phi_{1}}{\partial z}=0, \quad z=-h / R .
\end{aligned}
$$

Let $\phi_{1}=\left(\Phi_{1}(r, z, \tau) \mathrm{e}^{\mathrm{i} \Omega t}+\overline{\Phi_{1}}(r, z, \tau) \mathrm{e}^{-\mathrm{i} \Omega t}\right) \cos m \theta$, here $\overline{\Phi_{1}}$ denoted the complex conjugate of $\Phi_{1}, \Omega$ is called the natural frequency of the surface wave. Substituting $\phi_{1}$ into Eqs.(14)-(18), combining Eqs.(14)-(15), then $\Phi_{1}$ satisfies the following governing equation and boundary conditions:

$$
\begin{aligned}
& \frac{\partial^{2} \Phi_{1}}{\partial r^{2}}+\frac{1}{r} \frac{\partial \Phi_{1}}{\partial r}-\frac{m^{2}}{r^{2}} \Phi_{1}+\frac{\partial^{2} \Phi_{1}}{\partial z^{2}}=0, \quad 0<r<1, \quad-h / R<z<0 \\
& \frac{\partial \Phi_{1}}{\partial z}-\Omega^{2} \Phi_{1}+\frac{\Gamma}{\rho} \frac{\partial^{3} \Phi_{1}}{\partial z^{3}}=0, \quad z=0
\end{aligned}
$$




$$
\begin{array}{ll}
\frac{\partial \Phi_{1}}{\partial r}=0, & r=1, \\
\frac{\partial \Phi_{1}}{\partial z}=0, & z=-h / R .
\end{array}
$$

Let $\Phi_{1}=p(\tau) R(r) Z(z)$, substitute $\Phi_{1}$ into Eqs.(19)-(22), and solve this equation by separation of variables, then the first order velocity potential and the displacement of free surface are obtained as

$$
\begin{aligned}
& \phi_{1}=J_{m}(\lambda r) \cosh [\lambda(z+h / R)] \cdot\left[p(\tau) \mathrm{e}^{\mathrm{i} \Omega \mathrm{t}}+\bar{p}(\tau) \mathrm{e}^{-\mathrm{i} \Omega t}\right] \cos m \theta \\
& \eta_{1}=-\mathrm{i} \lambda / \Omega \cdot J_{m}(\lambda r) \sinh (\lambda h / R) \cdot\left[p(\tau) \mathrm{e}^{\mathrm{i} \Omega t}-\bar{p}(\tau) \mathrm{e}^{-\mathrm{i} \Omega t}\right] \cos m \theta
\end{aligned}
$$

The meaning of the above functions and symbols are the same as those in Ref.[8], and the following dispersion relationship is satisfied:

$$
\Omega^{2}=\lambda_{m n} \tanh \left(\lambda_{m n} h / R\right)\left(1+\Gamma / \rho \cdot \lambda_{m n}^{2}\right)=\Omega_{m n}^{2} .
$$

\section{Second Order Approximate Solutions}

The governing equation and boundary conditions of second order problem are

$$
\begin{aligned}
& \frac{\partial^{2} \phi_{2}}{\partial r^{2}}+\frac{1}{r} \frac{\partial \phi_{2}}{\partial r}+\frac{1}{r^{2}} \frac{\partial^{2} \phi_{2}}{\partial \theta^{2}}+\frac{\partial^{2} \phi_{2}}{\partial z^{2}}=0, \quad 0<r<1, \quad-h / R<z<0, \\
& \frac{\partial \phi_{2}}{\partial t}+\eta_{2}-\frac{\Gamma}{\rho}\left(\frac{\partial^{2} \eta_{2}}{\partial r^{2}}+\frac{1}{r} \frac{\partial \eta_{2}}{\partial r}+\frac{1}{r^{2}} \frac{\partial^{2} \eta_{2}}{\partial \theta^{2}}\right) \\
= & -\frac{\partial^{2} \phi_{1}}{\partial t \partial z} \cdot \eta_{1}-\frac{1}{2}\left[\left(\frac{\partial \phi_{1}}{\partial r}\right)^{2}+\frac{1}{r^{2}}\left(\frac{\partial \phi_{1}}{\partial \partial^{\prime}}\right)^{2}+\left(\frac{\partial \phi_{1}}{\partial z}\right)^{z j}\right], \quad z=0, \\
& \frac{\partial \eta_{2}}{\partial i}-\frac{\partial \phi_{2}}{\partial z}=-\frac{\partial \phi_{1}}{\partial r} \frac{\partial \eta_{1}}{\partial r}-\frac{1}{r^{2}} \frac{\partial \phi_{1}}{\partial \theta} \frac{\partial \eta_{1}}{\partial \theta}+\frac{\partial^{2} \phi_{1}}{\partial z^{2}} \eta_{1}, \quad z=0, \\
& \frac{\partial \phi_{2}}{\partial r}=0, \quad r=1, \\
& \frac{\partial \phi_{2}}{\partial z}=0, \quad z=-\frac{h}{R} .
\end{aligned}
$$

Combining boundary conditions (27) and (28), eliminating $\eta_{2}$, using the expressions (23) and (24) of first order solution, we can assume second order velocity potential $\phi_{2}$ has the form of

$$
\phi_{2}=B_{m, G_{l}}(r, z) \cos 2 m \theta \cdot\left(p^{2}(\tau) \mathrm{e}^{2 \mathrm{i} \Omega t}-\bar{p}^{2}(\tau) \mathrm{e}^{-2 \mathrm{i} \Omega t}\right),
$$

where $B_{m, G_{l}}(r, z)$ is the function of $r$ and $z$. Take Eq.(31) into Eqs.(26)-(30), then the function $B_{m, G_{l}}(r, z)$ satisfies the following governing equation and boundary conditions:

$$
\begin{aligned}
& \frac{\partial^{2} B}{\partial r^{2}}+\frac{1}{r} \frac{\partial B}{\partial r}-\frac{4 m^{2}}{r^{2}} B+\frac{\partial^{2} B}{\partial z^{2}}=0, \quad 0<r<1, \quad-h / R<z<0, \\
& \frac{\partial B}{\partial z}-4 \Omega^{2} B+\frac{\Gamma}{\rho} \frac{\partial^{3} B}{\partial z^{3}}=G_{l}(r), \quad z=0, \\
& \frac{\partial B}{\partial r}=0, \quad r=1, \\
& \frac{\partial B}{\partial z}=0, \quad z=-\frac{h}{R} .
\end{aligned}
$$


The expression of $B_{m, G_{l}}(r, z)$ can be obtained by solving Eqs.(32)-(35), then instituting $B_{m, G_{l}}(r, z)$ into $(31)$, We can obtain the second approximate solution of velocity potential $\phi_{2}$ :

$$
\begin{aligned}
& \phi_{2}(r, \theta, z, t, \tau)=\left[X_{1}(r, z)+X_{2}(r, z) \cos (2 m \theta)\right] \cdot\left[p^{2}(\tau) \mathrm{e}^{2 \mathrm{i} \Omega t}-\bar{p}^{2}(\tau) \mathrm{e}^{-2 \mathrm{i} \Omega t}\right] \\
& \eta_{2}(r, \theta, t, \tau)=\left[Y_{1}(r)+Y_{2}(r) \cos (2 m \theta)\right] \cdot\left[p^{2}(\tau) \mathrm{e}^{2 \mathrm{i} \Omega t}+\bar{p}^{2}(\tau) \mathrm{e}^{-2 \mathrm{i} \Omega t}\right] .
\end{aligned}
$$

The detailed expressions of $X_{1}(r, z), X_{2}(r, z), Y_{1}(r)$ and $Y_{2}(r)$ are given in Ref.[10]. We have solved the second order velocity potential and the displacement of free surface. However, the slowly varying amplitude $p(\tau)$ is still not determined. We continue to consider the third order governing equation and the boundary conditions. Amplitude equation of $p(\tau)$ will be derived using the solvability condition of the third problem.

\section{Third Order Approximation Problems and Amplitude Equation}

The governing equation and boundary conditions of third order problem are

$$
\begin{aligned}
& \frac{\partial^{2} \phi_{3}}{\partial r^{2}}+\frac{1}{r} \frac{\partial \phi_{3}}{\partial r}+\frac{1}{r^{2}} \frac{\partial^{2} \phi_{3}}{\partial \theta^{2}}+\frac{\partial^{2} \phi_{3}}{\partial z^{2}}=0, \quad 0<r<1, \quad-h / R<z<0, \\
& \frac{\partial \phi_{3}}{\partial t}+\eta_{3}-\frac{\Gamma}{\rho} \cdot\left(\frac{\partial^{2} \eta_{3}}{\partial r^{2}}+\frac{1}{r} \cdot \frac{\partial \eta_{3}}{\partial r}+\frac{1}{r^{2}} \cdot \frac{\partial^{2} \eta_{3}}{\partial \theta^{2}}\right) \\
& =\frac{1}{2} \frac{\partial^{3} \phi_{1}}{\partial z^{2} \partial t} \eta_{1}^{2}-\frac{\partial \phi_{1}}{\partial \tau}-\frac{\partial^{2} \phi_{2}}{\partial t \partial z} \eta_{1}-\frac{\partial^{2} \phi_{1}}{\partial t \partial z} \eta_{2}-\frac{\partial \phi_{1}}{\partial r} \frac{\partial \phi_{2}}{\partial r}-\frac{\partial \phi_{1}}{\partial r} \frac{\partial^{2} \phi_{1}}{\partial r \cdot \partial z} \eta_{1} \\
& -\frac{\partial \phi_{1}}{\partial z} \frac{\partial \phi_{2}}{\partial z}-\frac{1}{r^{2}}\left(\frac{\partial \phi_{1}}{\partial \theta} \frac{\partial \phi_{2}}{\partial \theta}+\frac{\partial \phi_{1}}{\partial \theta} \frac{\partial^{2} \phi_{1}}{\partial \partial^{2} z} \eta_{\mathrm{i}}\right)-\frac{\partial \phi_{1}}{\partial z} \frac{\partial^{2}}{\partial z^{2}} \frac{\phi_{1}}{\partial{ }^{2}} \eta_{1} \\
& -\frac{1}{r^{2}}\left(\frac{\partial \phi_{1}}{\partial \hat{\sigma}} \frac{\partial \phi_{2}}{\partial \theta}+\frac{\delta \phi_{1}}{\partial \hat{b}} \frac{\partial^{2} \phi_{1}}{\partial \bar{\theta} \dot{\partial} \partial z} \eta_{1}\right)-i_{1} \cos \left(2 \omega_{0} t\right), \quad z=0 \\
& \frac{\phi_{3}}{\partial z}-\frac{\partial \eta_{3}}{\partial t} \\
& =-\frac{\partial^{2} \phi_{2}}{\partial z^{2}} \eta_{1}-\frac{\partial^{2} \phi_{1}}{\partial z^{2}} \eta_{2}-\frac{1}{2} \frac{\partial^{3} \phi_{1}}{\partial z^{3}} \eta_{1}^{2}+\frac{\partial \eta_{1}}{\partial \tau}+\frac{\partial \eta_{2}}{\partial r} \frac{\partial \phi_{1}}{\partial r}+\frac{\partial \eta_{1}}{\partial r} \frac{\partial \phi_{2}}{\partial r} \\
& +\frac{\partial \eta_{1}}{\partial r} \eta_{1} \frac{\partial^{2} \phi_{1}}{\partial r \partial z}+\frac{1}{r^{2}}\left(\frac{\partial \eta_{1}}{\partial \theta} \frac{\partial \phi_{2}}{\partial \theta}+\frac{\partial \eta_{2}}{\partial \theta} \frac{\partial \phi_{1}}{\partial \theta}+\frac{\partial \eta_{1}}{\partial \theta} \frac{\partial^{2} \phi_{1}}{\partial \theta \partial z} \eta_{1}\right), \quad z=0 \\
& \frac{\partial \phi_{3}}{\partial r}=0, \quad r=1 \text {, } \\
& \frac{\partial \phi_{3}}{\partial z}=0, \quad z=-\frac{h}{R} .
\end{aligned}
$$

Applying the same method as Ref.[8], the amplitude equations of free surface wave associated with slowly variable time are

$$
\begin{aligned}
& \frac{d p_{1}(\tau)}{d \tau}=M_{1} p_{2}(\tau)\left[p_{1}^{2}(\tau)+p_{2}^{2}(\tau)\right]+M_{2}\left[p_{1}(\tau) \sin (2 \sigma \tau)-p_{2}(\tau) \cos (2 \sigma \tau)\right] \\
& \frac{d p_{2}(\tau)}{d \tau}=-M_{1} p_{1}(\tau)\left[p_{1}^{2}(\tau)+p_{2}^{2}(\tau)\right]-M_{2}\left[p_{1}(\tau) \cos (2 \sigma \tau)+p_{2}(\tau) \sin (2 \sigma \tau)\right]
\end{aligned}
$$

All parameters and their detailed expressions in Eqs.(43) and (44) were given in Ref.[10].

\section{Computational Results}

\subsection{Influence of surface tension on mode selection}

In order to demonstrate the influence of surface tension on the natural frequency and mode selection, we illustrated the variation of the dimensional natural frequency with the wave number 
both with surface tension and without surface tension in Fig.1. The selection of parameters is similar to those used in Refs.[5-7]. It can be shown that the frequency of the surface wave increases with the increase of the wave number no matter the surface tension was included or not. When forced frequency is low (e.g., smaller than $10 \mathrm{~Hz}$ ), the effect of surface tension on mode selection can be ignored. However,

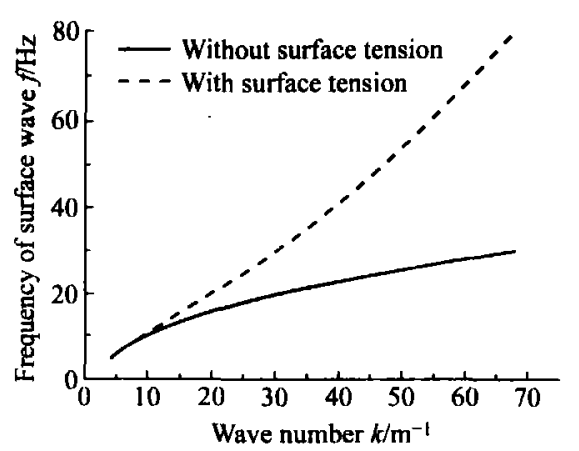

Fig.1 Variation of dimensional natural frequency with wave number (depth of fluid $h=1.0 \mathrm{~cm}$, radius of the vessel $R=7.5 \mathrm{~cm}$, surface tension $\Gamma=$ $0.0727 \mathrm{~N} / \mathrm{m}$, density of fluid $\rho=10^{3}$ $\mathrm{kg} / \mathrm{m}^{3}$ )

\subsection{Comparison with experisrent}

A comparison can also be naie beiween the iheoretical and laboratory contours of Refs. [6-8]

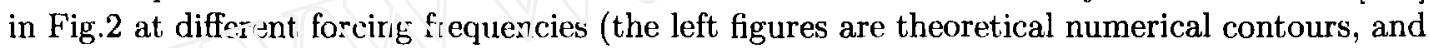
the right ones ar experimental contours of the surface flow patterns). The solid and dashed lines denote the position of free surface above and below the equilibrium surface, respectively. When the effect of surface tension is taken into account, the theoretical result closes to experimental ones better than that of no surface tension. However, the discrepancy of forced frequency is still very large. The reasons are possibly the effect of contact line, the mode competition and the viscosity of the fluid, etc. The influence of the weak viscosity on the mode selection will be discussed in another paper.

\section{Conclusions}

From above analysis, following results can be obtained:

(1) The method of two-time scale expansion is effective to resolve the problem with the effect of surface tension in vertically oscillating circular cylindrical container.

(2) The nonlinear amplitude equations (43)-(44) and free surface displacement (13) can be correctly used to describe the surface wave motion in a vertically excited vessel.

(3) When the wave number is small, the influence of the surface tension on pattern selection is insignificant. However, when the wave number is large, the effect of the surface tension is important.

(4) The surface tension has the function, which causes free surface returning to equilibrium location. That is to say, it needs higher forced frequency to produce the same flowing pattern incorporating surface tension than that of having no surface tension. 

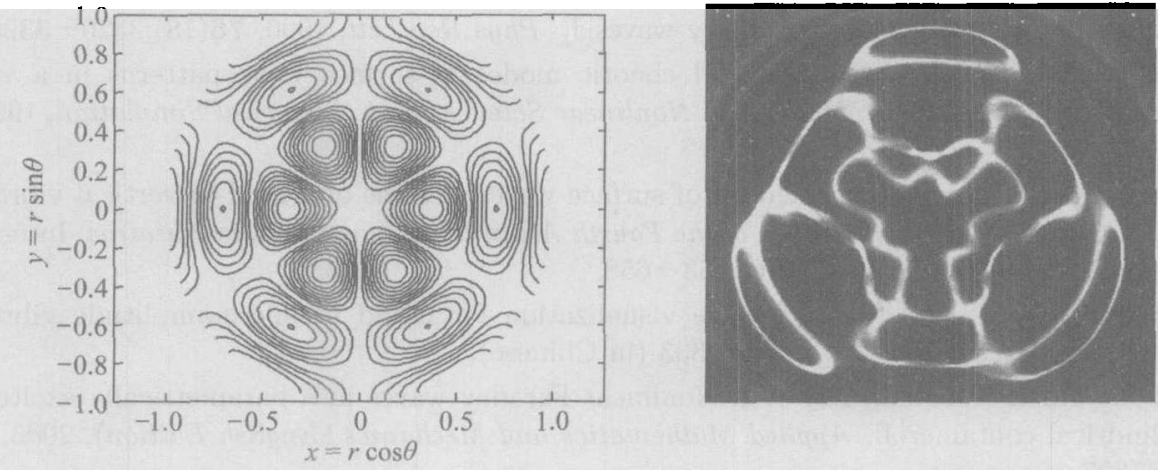

(a) $(3,3)$ mode (left, forced frequency $f=12.63 \mathrm{~Hz}$; right, forced frequency $f=20 \mathrm{~Hz}$ )
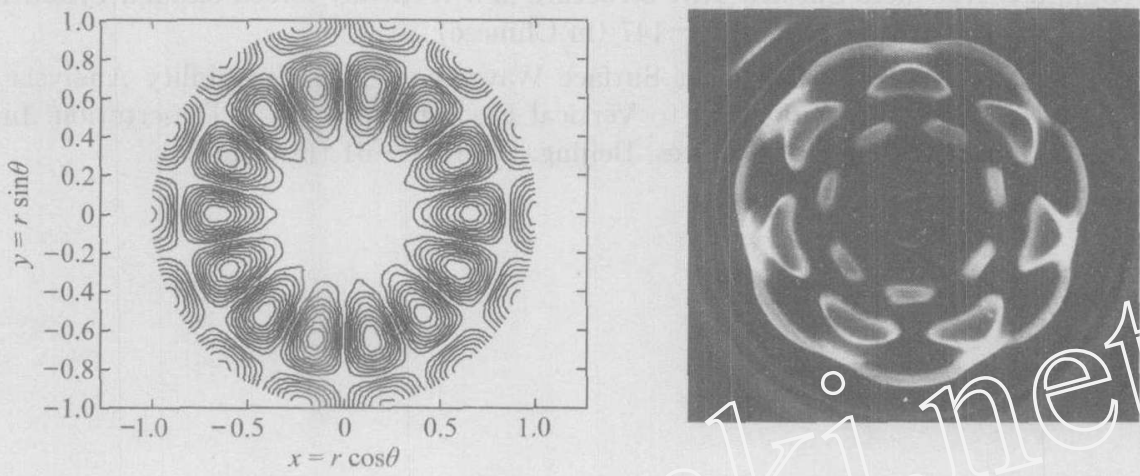

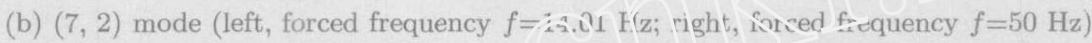
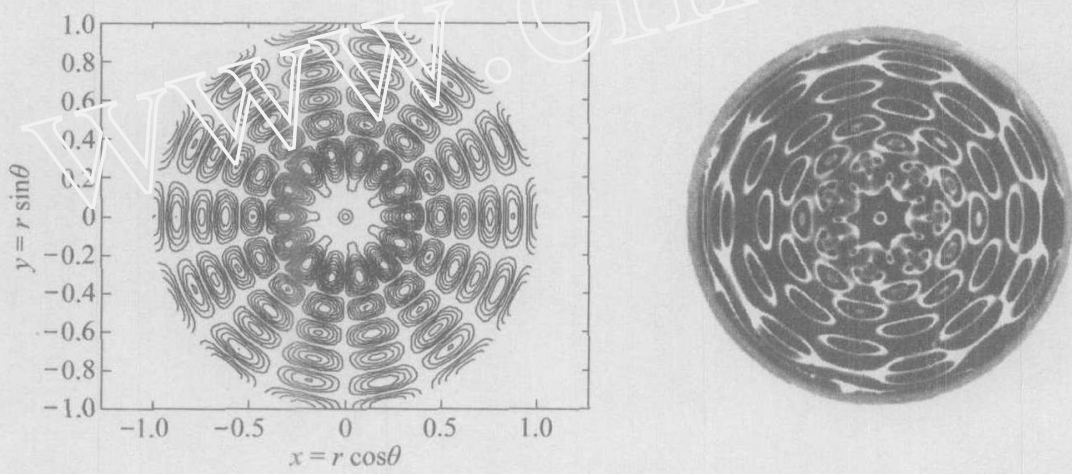

(c) $(7,6)$ mode (left, forced frequency $f=26.02 \mathrm{~Hz}$; right, forced frequency $f=52 \mathrm{~Hz}$ )

Fig.2 Comparison of theoretical contours of surface wave mode with those of experiment (depth of fluid $h=1.0 \mathrm{~cm}$, radius of the vessel $R=7.5 \mathrm{~cm}$, the forcing amplitude $A=11.4 \mu \mathrm{m}$, surface tension coefficient $\Gamma=0.0727 \mathrm{~N} / \mathrm{m}$, density of fluid $\rho=10^{3} \mathrm{~kg} / \mathrm{m}^{3}$ )

\section{References}

[1] Zhang W, Viñal J. Pattern formation in weakly damped parametric surface waves[J]. J Fluid Mech, 1997, 336(7):301-330.

[2] Ciliberto S, Gollub J P. Chaotic mode competition in parametrically forced surface waves[J]. $J$ Fluid Mech, 1985, 158(17):381-398.

[3] Kudrolli A, Gollub J P. Pattern and spatiotemporal chaos in parametrically forced surface waves: a systematic survey at large aspect ratio[J]. Physica $D, 1996,97(1): 113-154$. 
[4] Pushkarev A N. Turbulence of capillary waves[J]. Phys Rev Lett, 1996, 76(18):3320-3323.

[5] E Xuequan, Gao Yuxin. Ordered and chaotic modes of surface wave patterns in a vertically oscillating fluid[J]. Communications in Nonlinear Sciences and Numerical Simulation, 1996, 1(2): $1-6$.

[6] E Xuequan, Gao Yuxin. Visualization of surface wave patterns of a fluid in vertical vibration[C]. In: Wei Qingding (ed). Proceedings of the Fourth Asian Symposium on Visualization. International Academic Publishers, Beijing, 1996, 653-658.

[7] Gao Yuxin, E Xuequan. Surface wave visualization for liquid in micro-amplitude vibration[J]. Experimental Mech, 1998, 13(3):326-333 (in Chinese).

[8] Jian Yongjun, E Xuequan, Bai Wei. Nonlinear Faraday waves in a parametrically excited circular cylindrical container[J]. Applied Mathematics and Mechanics (English Edition), 2003, 24(10): $1194-1207$.

[9] Jian Yongjun, E Xuequan. Surface wave structure in a vertically forced circular cylindrical vessel [J]. $J$ Hydrodynamics, 2003, 18(2):135-147 (in Chinese).

[10] Jian Yongjun. Study on the Nonlinear Surface Waves and Their Instability Analysis in a Circular Cylindrical Container Subjected to Vertical Excitation[D]. Ph D Dissertation. Institute of Mechanics, Chinese Academy of Sciences, Beijing, 2003, 50-61 (in Chinese). 